\title{
Convex combination of grouping functions for image thresholding. Selection of weighting vectors
}

\author{
Aranzazu Jurio ${ }^{1}$ Miguel Pagola $^{1}$ Daniel Paternain ${ }^{1}$ Nicolas Madrid $^{2}$ Humberto Bustince $^{1}$ \\ ${ }^{1}$ Departamento de Automatica y Computacion, Universidad Publica de Navarra \\ 31006 Pamplona, Spain (e-mail aranzazu.jurio@unavarra.es) \\ ${ }^{2}$ Centre of Excellence IT4Innovations - Division of University of Ostrava - IRAFM \\ Ostrava, Czech Republic
}

\begin{abstract}
In this work we present a thresholding algorithm for greyscale images. Our proposal is based on the use of grouping functions to find the best threshold. These functions are able to measure the membership of a grey intensity to the background or to the object of the image, so the best threshold is the one associated with the highest grouping value. To avoid the hard task concerning the choice of a suitable grouping function for any given image, we propose to use a combination of several of them. We study different ways of choosing the weights for this combination.
\end{abstract}

Keywords: Thresholding, grouping function, convex combination.

\section{Introduction}

One of the most used techniques in image segmentation is thresholding or segmentation by greylevels $[12],[13],[11]$. Such approaches are based on the assumption that different objects of the image are characterized just by the intensity of their pixels. This technique consists in finding a threshold $t$ such that the pixels whose intensities are lower or equal to $t$ belong to the background of the image while the intensities that are greater than $t$ belong to the object, or vice versa [4]. Comparing with other segmentation algorithms, the advantages of thresholding are the simplicity and low computational cost. This is why this procedure is commonly used as a first step of more complex segmentation algorithms.

In this work we present a new thresholding algorithm, generalizing previous fuzzy approaches [4], [8]. Our proposal is based on the construction, for every possible grey intensity, of two fuzzy sets $\left(Q_{B_{t}}\right.$ and $Q_{O_{t}}$ ) representing the belongingness of every greylevel to the background $\left(\mu_{Q_{B_{t}}}(q)\right)$ and to the object $\left(\mu_{Q_{O_{t}}}(q)\right)$ of the image respectively. The goal is to find the threshold for which the membership of every grey intensity to the object or to the background is maximum $\left(\mu_{Q_{B_{t}}}=1\right.$ or $\left.\mu_{Q_{O_{t}}}=1\right)$, so we are completely sure that those pixels belong to the background or to the object of the image.
To quantize the membership to any of two sets we use the concept of grouping. Any grouping function is a bivariate aggregation function that gets the maximum value if and only if one of the arguments is equal to 1 . In this work we study the axiomatization of these functions, propose some construction methods and relate grouping functions with overlap functions [5].

One of the difficulties of fuzzy thresholding approaches in the literature is how to choose the apropiate expression for any image. For example, if the thresholding is based on maximizing the similarity [4], the obtained results vary strongly according to the expression of similarity chosen. Hence, the choice of a suitable expression for a given image seems to be crucial in such approaches. To solve this problem, in this work we study that the convex combination of grouping functions is also a grouping function. Following this result, we can use several grouping expressions and combine them if we do not know a priori which is the appropiate one.

We also show an illustrative example for a medical imaging application, where we have to segment some magnetic resonance images (MRI). The purpose is to separate the gray matter from the white matter of a brain, which is a very helpful process to evaluate some diseases like Alzheimer or schizophrenia.

The rest of the contribution is organized in the following way. We start by recalling some preliminary concepts in Section 2. In Section 3 we study grouping functions, their relations with overlap functions and some construction methods. In Section 4 we present our image thresholding algorithm, and in Section 5 we show an illustrative example. We finish with some conclusions in Section 6.

\section{Preliminaries}

A strict negation [14] is a continuous and strictly decreasing function $N:[0,1]^{2} \rightarrow[0,1]$ such that $N(0)=1$ and $N(1)=0$. A strong negation is a strict negation that is also involutive, it means, $N(N(x))=x$ for all $x \in[0,1]$.

A triangular norm (t-norm) is a symmetric 
and associative bivariate aggregation function $T$ : $[0,1]^{2} \rightarrow[0,1]$ such that $T(x, 1)=x$ for all $x \in$ $[0,1]$. Some examples of t-norms are minimum function $T_{M}(x, y)=\min (x, y)$ or product function $T_{P}(x, y)=x \cdot y$. A triangular conorm (t-conorm) is a symmetric and associative bivariate aggregation function $S:[0,1]^{2} \rightarrow[0,1]$ such that $S(x, 0)=x$ for all $x \in[0,1]$. Some examples of t-conorms are the maximum function $S_{M}(x, y)=\max (x, y)$ or the probabilistic sum function $S_{P}(x, y)=x+y-x \cdot y$ $[2,9,1]$.

In this work we use restricted equivalence functions $(R E F)$ to build the fuzzy sets associated with an image [3], [4].

Definition 1 A function REF $:[0,1]^{2} \rightarrow[0,1]$ is called restricted equivalence function if it satisfies the following conditions:

1. $R E F(x, y)=R E F(y, x)$ for all $x, y \in[0,1]$;

2. $R E F(x, y)=1$ if and only if $x=y$;

3. $\operatorname{REF}(x, y)=0$ if and only if $x=1$ and $y=0$ or $x=0$ and $y=1$;

4. $\operatorname{REF}(x, y)=\operatorname{REF}(N(x), N(y))$ for all $x, y \in$ $[0,1]$, being $N$ a strong negation;

5. if $x \leq y \leq z$ then $\operatorname{REF}(x, y) \geq \operatorname{REF}(x, z)$ and $R E F(y, z) \geq R E F(x, z)$, for all $x, y, z \in[0,1]$.

We use two different concepts over the weighting vectors $\left(W=\left\{w_{1}, \ldots, w_{n}\right\}\right)[15]$. The first one is the orness, which measures the degree to which the aggregation is like an or operator:

$$
\operatorname{orness}(W)=\frac{1}{n-1} \sum_{i=1}^{n}(n-i) w_{i} .
$$

The second concept, called dispersion, measures the degree to which $\mathrm{W}$ takes into account all information in the aggregation:

$$
\operatorname{disp}(W)=-\sum_{i=1}^{n} w_{i} \cdot \ln w_{i}
$$

\section{Grouping functions}

We start by recalling the definition of grouping function. Observe that a grouping function is a particular case of binary aggregation.

Definition 2 [6] A function $G_{G}:[0,1]^{2} \rightarrow[0,1]$ is a grouping function if it satisfies the following conditions:

$\left(G_{G} 1\right) G_{G}(x, y)=G_{G}(y, x)$ for all $x, y \in[0,1]$;

$\left(G_{G} 2\right) G_{G}(x, y)=0$ if and only if $x=y=0$;

$\left(G_{G} 3\right) G_{G}(x, y)=1$ if and only if $x=1$ or $y=1$;

$\left(G_{G} 4\right) G_{G}$ is non-decreasing;

$\left(G_{G} 5\right) G_{G}$ is continuous.

A relation of duality between grouping functions and overlap functions is given in [5]. We use this relation to present several construction methods of grouping functions.

\subsection{Overlap functions}

Definition 3 [5] A function $G_{O}:[0,1]^{2} \rightarrow[0,1]$ is an overlap function if it satisfies the following properties:

( $\left.G_{O} 1\right) G_{O}$ is symmetric.

$\left(G_{O} 2\right) G_{O}(x, y)=0$ if and only if $x y=0$.

$\left(G_{O} 3\right) G_{O}(x, y)=1$ if and only if $x y=1$.

$\left(G_{O} 4\right) G_{O}$ is non-decreasing.

$\left(G_{O} 5\right) G_{O}$ is continuous.

Theorem 1 Let $G_{O}$ be an overlap function and let $N$ be a strict negation. Then

$$
G_{G}(x, y)=N\left(G_{O}(N(x), N(y))\right)
$$

is a grouping function. Reciprocally, we have that

$$
G_{O}(x, y)=N\left(G_{G}(N(x), N(y))\right)
$$

is an overlap function.

Proof $1\left(G_{G} 1\right), \quad\left(G_{G} 4\right)$ and $\left(G_{G} 5\right)$ are direct. $\left(G_{G} 2\right) G_{G}(x, y)=0=N\left(G_{O}(N(x), N(y))\right)$ if and only if $G_{O}(N(x), N(y))=1$ if and only if $N(x)=$ $N(y)=1$ if and only if $x=y=0 . \quad\left(G_{G} 3\right)$, $G_{G}(x, y)=1=N\left(G_{O}(N(x) ; N(y))\right)$ if and only if $G_{O}(N(x), N(y))=0$ if and only if $N(x)=0$ or $N(y)=0$ if and only if $x=1$ or $y=1$.

Following the relation between overlap functions and t-norms, in this work we proof that any associative grouping function is also a t-conorm. However, the reciprocal of this theorem does not hold, as it is clear if we consider any non-continuous t-conorm.

Theorem 2 Let $G_{G}$ be an associative grouping function. Then $G_{G}$ is a t-conorm.

Proof 2 We just need to proof that 0 is the neutral element of $G_{G}$. Because of the continuity of $G_{G}$ and $G_{G}(0,1)=1$ and $G_{G}(0,0)=0$, we can say that for any $x \in] 0,1[$ there exists a $y \in] 0,1[$ such that $x=G_{G}(y, 0)$. Then $G_{G}(x, 0)=G_{G}\left(G_{G}(y, 0), 0\right)=$ $G_{G}\left(y, G_{G}(0,0)\right)=G_{G}(y, 0)=x$ and in a similar way $G_{G}(0, x)=x$.

Example 1 An associative grouping function and therefore a $t$-conorm is the maximum function.

$$
G_{G}(x, y)=\max (x, y)
$$

\subsection{Convex combination}

In Theorem 3 we show the main improvement of this work to the fuzzy thresholding methods present in the literature. We introduce how to obtain a new grouping function from several expressions. This result helps us to present an algorithm where we do not need to know which is the most suitable expression for every image a priori. 
Theorem 3 Let $G_{G} 1, \ldots, G_{G} m$ be $m$ grouping functions and let $w_{1}, \ldots, w_{m}$ be $m$ non-negative weights such that $\sum_{i=1}^{m} w_{i}=1$. Then the convex sum $G_{G}:[0,1]^{2} \rightarrow[0,1]$ given by

$$
G_{G}(x, y)=\sum_{i=1}^{m} w_{i} G_{G} i(x, y)
$$

is a grouping function.

\section{Proof 3 Direct.}

\subsection{Construction of grouping functions}

The first result on this section is a representation theorem of grouping functions.

Theorem 4 The function $G_{G}:[0,1]^{2} \rightarrow[0,1]$ is a grouping function if and only if

$$
G_{G}(x, y)=\frac{f(x, y)}{f(x, y)+h(x, y)}
$$

for $f, h:[0,1]^{2} \rightarrow[0,1]$ such that

1. $f$ and $h$ are symmetric;

2. $f$ is non-decreasing and $h$ is non-increasing;

3. $f(x, y)=0$ if and only if $x=y=0$;

4. $h(x, y)=0$ if and only if $x=1$ or $y=1$;

5. $f y h$ are continuous functions.

Proof 4 We have to take into account that $f(x, y)+h(x, y) \neq 0$ for all $(x, y) \in[0,1]^{2}$. Then the necessity is straightaway taking $f(x, y)=G_{G}(x, y)$ and $h(x, y)=1-G_{G}(x, y)$. (Sufficiency) $\left(G_{G} 1\right),\left(G_{G} 2\right),\left(G_{G} 3\right)$ and $\left(G_{G} 5\right)$ are direct. $\left(G_{G} 4\right)$ If $x_{1} \leq x_{2}$ then $f\left(x_{1}, y\right) \leq f\left(x_{2}, y\right)$ and $h\left(x_{2}, y\right) \leq h\left(x_{1}, y\right)$. So we have that $f\left(x_{1}, y\right) h\left(x_{2}, y\right) \leq f\left(x_{2}, y\right) h\left(x_{1}, y\right)$. Adding $f\left(x_{1}, y\right) f\left(x_{2}, y\right)$ to both sides of the inequality we have $f\left(x_{1}, y\right) f\left(x_{2}, y\right)+f\left(x_{1}, y\right) h\left(x_{2}, y\right) \leq$ $f\left(x_{1}, y\right) f\left(x_{2}, y\right)+f\left(x_{2}, y\right) h\left(x_{1}, y\right)$. We can rewrite $G_{G}\left(x_{1}, y\right)=\frac{f\left(x_{1}, y\right)}{f\left(x_{1}, y\right)+h\left(x_{1}, y\right)} \leq \frac{f\left(x_{2}, y\right)}{f\left(x_{2}, y\right)+h\left(x_{2}, y\right)}=$ $G_{G}\left(x_{2}, y\right)$.

The theorem above allows us to construct several grouping function. Some of them are shown in Examples 2-4.

Example 2 If we take $f(x, y)=\max (x, y)$ and $h(x, y)=\sqrt{(1-x)(1-y)}$ we have

$$
G_{G}(x, y)=\frac{\max (x, y)}{\max (x, y)+\sqrt{(1-x)(1-y)}}
$$

Example 3 If we take $f(x, y)=\max (x, y)$ and $h(x, y)=(1-x)(1-y)$ we have

$$
G_{G}(x, y)=\frac{\max (x, y)}{\max (x, y)+(1-x)(1-y)}
$$

Example 4 If we take $f(x, y)=1-$ $\sqrt{(1-x)(1-y)}$ and $h(x, y)=\min ((1-x),(1-y))$ we have

$$
G_{G}(x, y)=\frac{1-\sqrt{(1-x)(1-y)}}{1-\sqrt{(1-x)(1-y)}+\min ((1-x),(1-y))}
$$

We can also obtain new grouping expressions from existing ones. In Theorem 5 we show a construction method based on a grouping function and two continuous monotone mappings.

Theorem 5 Let $\varphi_{1}, \varphi_{2}:[0,1] \rightarrow[0,1]$ two continuous monotone mappings such that $\varphi_{1}(x)=0$ if and only if $x=0, \varphi_{1}(x)=1$ if and only if $x=1$ and $\varphi_{2}(x)=0$ if and only if $x=0, \varphi_{2}(x)=1$ if and only if $x=1$. Let $G_{G}:[0,1]^{2} \rightarrow[0,1]$ be a grouping function. Then, the mapping $G_{G}^{\varphi_{1}, \varphi_{2}}$ defined as

$$
G_{G}^{\varphi_{1}, \varphi_{2}}(x, y)=\varphi_{1}\left(G_{G}\left(\varphi_{2}(x), \varphi_{2}(y)\right)\right)
$$

is also a grouping function.

\section{Proof 5 Direct.}

Example 5 Consider the grouping function $G_{G}(x, y)=\max (x, y)$. Let $p=q=2$ and consider the following mappings: $\varphi_{1}(x)=x^{p}$ and $\varphi_{2}(x)=x^{q}$. Then the mapping

$$
G_{G}^{\varphi_{1}, \varphi_{2}}(x, y)=\left(\max \left(x^{2}, y^{2}\right)\right)^{2}
$$

is also a grouping function.

\section{Thresholding algorithm based on grouping functions}

In this work we propose the use of grouping functions as the metric to calculate the optimal threshold of an image. We construct, for every greylevel, a fuzzy set associated with the background and a fuzzy set associated with the object of the image. In this case, we select as the best threshold the one associated with the greatest degree of grouping between both fuzzy sets. Hence we obtain that each intensity has a large membership degree either to the background or to the object, it means, every intensity present in the image is very close to the average intensity of the background or to the average intensity of the object. To avoid the selection of a suitable grouping expression for every image, which is a very difficult task, we use the convex combination of several expressions. Note that as exposed in Theorem 3, the convex combination of grouping functions results in a new grouping function. This step allows the algorithm to manage a consensus between the results obtained by several grouping expressions. The scheme of our proposal is shown in Algorithm 1. 


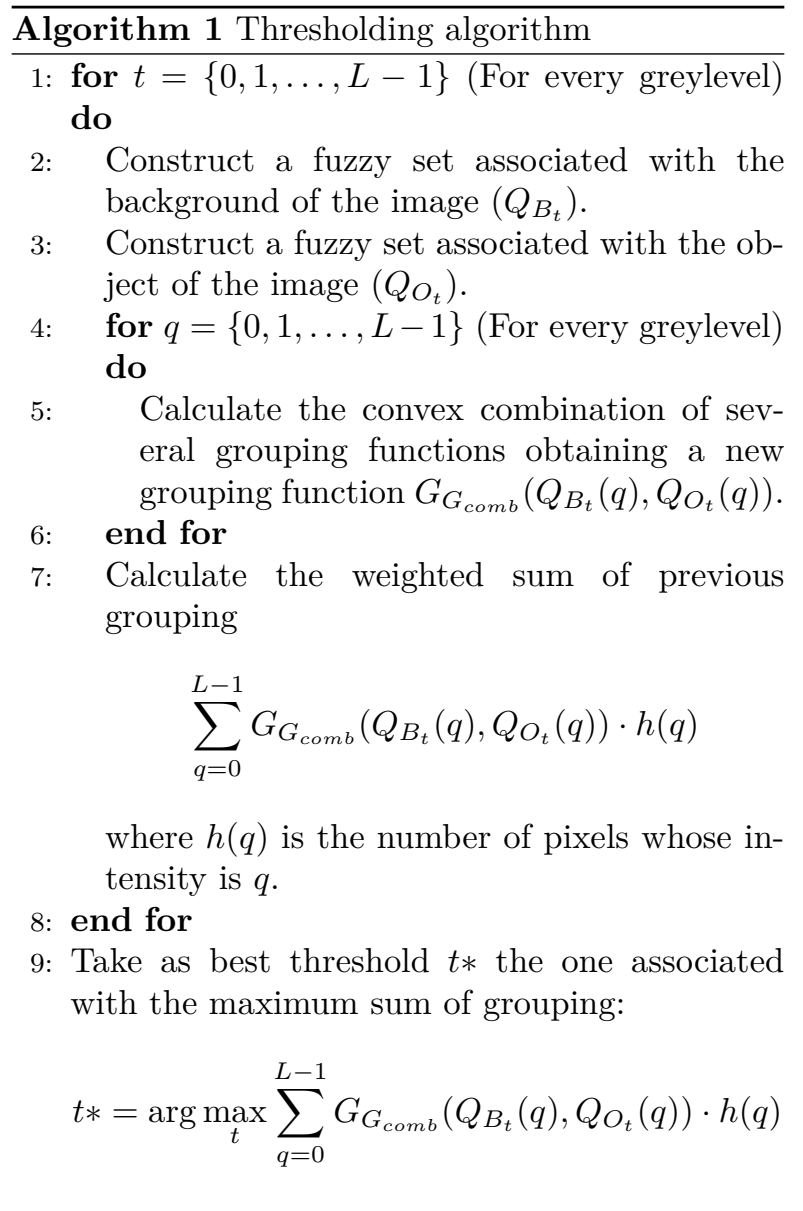

\subsection{Construction of fuzzy sets associated with the image}

In thresholding problems with only one threshold, it is assumed that the image is divided into two areas, so the procedure consists just in separating one object from the background. Based on the study presented in [4], in this work we construct two fuzzy sets $\left(Q_{B_{t}}\right.$ associated with the background and $Q_{O_{t}}$ associated with the object) from restricted equivalence functions, bearing in mind the following reasoning: the more similar the greylevel $(q)$ to the average of the background (resp. of the object) intensities the greater the membership of $q$ to the fuzzy set associated with the background (resp. to the object).

For a fixed greylevel $t$, we start by calculating the average value of the intensities belonging to the background $\left(m_{B}(t)\right)$ and to the object $\left(m_{O}(t)\right)$ using the following expressions:

$$
m_{B}(t)=\frac{\sum_{q=0}^{t} q \cdot h(q)}{\sum_{q=0}^{t} h(q)} m_{O}(t)=\frac{\sum_{q=t+1}^{L-1} q \cdot h(q)}{\sum_{q=t+1}^{L-1} h(q)}
$$

where $q(h)$ is the number of pixels whose intensity is $q$.

Let $R E F$ be a restrictred equivalence function, we construct the fuzzy sets $Q_{B_{t}}$ and $Q_{O_{t}}$ with the following membership functions, for every greylevel

$$
\begin{aligned}
q=0,1, \ldots, L-1: & \\
\mu_{Q_{B_{t}}}(q) & =\operatorname{REF}\left(\frac{q}{L-1}, \frac{m_{B}(t)}{L-1}\right) \\
\mu_{Q_{O_{t}}}(q) & =\operatorname{REF}\left(\frac{q}{L-1}, \frac{m_{O}(t)}{L-1}\right)
\end{aligned}
$$

Note that, with this construction method and due to property (2) of Definition 1, a greylevel has the greatest membership degree to the background (object) fuzzy set only if its intensity is the same as the average intensities of the background (object) of the image. That is:

- $\mu_{Q_{B_{t}}}(q)=1$ if and only if $q=m_{B}(t)$.

- $\mu_{Q_{O_{t}}}(q)=1$ if and only if $q=m_{O}(t)$.

\subsection{Grouping calculus}

To calculate the grouping value associated with each possible threshold $t$, we use $n$ different grouping functions $\left(G_{G} 1, G_{G} 2, \ldots, G_{G} n\right)$. A grouping function takes two arguments and calculates the group level between both of them. In this case we compute the grouping, for every greylevel, between the membership degree to the fuzzy set associated with the background and the membership degree to the fuzzy set associated with the object.

Using the result obtained in Theorem 3, we combine the $n$ grouping functions prevously calculated. In this way, we obtain a new grouping function that, experimentally, it outperforms the result obtained by the worst grouping expression selected. This step helps us to solve the problem of choosing a grouping expression not suitable for a specific image, what finishes in wrong results.

Once we have one sole value for the grouping of every greylevel, we calculate the sum. This is the value for the grouping associated with the threshold $t$.

\subsection{Selection of the maximum grouping}

Every possible threshold $t=\{0,1, \ldots, L-1\}$ has a grouping value associated with it, calculated as the sum of the grouping function in several points. To get the best threshold, we choose the one associated with the highest grouping value. We choose the maximum value because of grouping functions properties. Specifically, the sum is maximum if $G_{G}\left(\mu_{B_{t}}(q), \mu_{O_{t}}(q)\right)=1$ for all $q=\{0,1, \ldots, L-1\}$. By property $\left(G_{G} 2\right)$ of Definition 2 this is achieved in two cases:

- $\mu_{B_{t}}(q)=1$, so $q=m_{B}(t)$. In this case we are completely sure that the pixels whose intensity is $q$ belong to the background of the image, because this intensity is exactly the average intensity of all the pixels of the background.

- $\mu_{O_{t}}(q)=1$, so $q=m_{O}(t)$. In this case we are completely sure that the pixels whose intensity is $q$ belong to the object of the image, because 
this intensity is exactly the average intensity of all the pixels of the object.

In this sense, by choosing the highest grouping value we select the threshold for which all the pixels whose intensity is lower than the threshold are very closed to the average of background (object) intensities and all the pixels whose intensity is greater than the threshold are very closed to the average intensity of the object (background).

\section{Illustrative example}

In this section we show the performance of the proposed algorithm over 10 T1-weighted magnetic resonance images (see Figure 1). These images are provided by the Center for Morphometric Analysis at Massachusetts General Hospital (available at http://www.cma.mgh.harvard.edu/ibsr/). The aim of the segmentation of this kind of images is to separate each of the pixels inside the brain into one of the following two types: grey matter and white matter. This segmentation can be viewed as a first step of a volumetric analysis of the brain regions, which is very useful to evaluate the evolution of diseases such as Alzheimer, epilepsy or schizophrenia $[7,10]$. To measure the quality of the segmented results, we compare them with an ideal handmade segmentation provided at the same webpage (see Figure 2). For this illustrative example we make this comparison by the percentage of well classified pixels because of its simplicity, although there exist more advance measures like Dice and Jaccard coefficients.

In this example we use seven grouping functions for the step 5 of the algorithm:

- $G_{G} 1(x, y)=\max (x, y)$

- $G_{G} 2(x, y)=\frac{\max (x, y)}{\max (x, y)+\sqrt{(1-x)(1-y)}}$

- $G_{G} 3(x, y)=1-\sqrt{(1-x)(1-y)}$

- $G_{G} 4(x, y)=x+y-x y$

- $G_{G} 5(x, y)=\frac{2-(1-x)^{2}(1-y)-(1-x)(1-y)^{2}}{2}$

- $G_{G} 6(x, y)=\left(\max \left(x^{2}, y^{2}\right)\right)^{2}$

- $G_{G} 7(x, y)=\sqrt{x^{2}+y^{2}-x^{2} y^{2}}$

To calculate the convex combination of grouping functions we do not know a priori which expressions are more suitable for this kind of images. In this scenario, the first approach we can execute is to assign the same weight to every expression. In this example, we have seven expressions, so the weight for each of them should be $1 / 7$. But we can also use different weights for any expression. In this work we use OWA operators [15], so we have to arrange the grouping values for any possible greylevel. We compare three different ideas to assign the weights of the OWA operator, depending on their orness. For any of them, we use a singleton (only one expression has a weight maximum and the rest of the weights are equal to zero, so the dispersion is 0 ) and a weighting vector derived from a linguistic quantifier [16]. In this sense, if we want to highlight the grouping expressions with large orness we use the weighting vectors:

- Max: $\left[\begin{array}{lllllll}1 & 0 & 0 & 0 & 0 & 0 & 0\end{array}\right]$

- Half: [l $\left.\begin{array}{lllllll}0.2857 & 0.2857 & 0.2857 & 0.1429 & 0 & 0 & 0\end{array}\right]$

where the second one is derived from the expression "At least half". For vectors with a medium orness value we use the weighting vectors:

- Med: [0 $\left.0 \begin{array}{llllll}0 & 0 & 1 & 0 & 0 & 0\end{array}\right]$

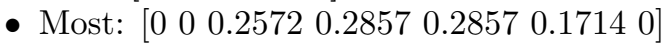

where the second one is derived from the expression "Most". Finally, if we want to highlight the the grouping expressions with low orness, we use the weighting vectors:

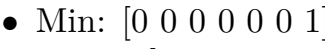

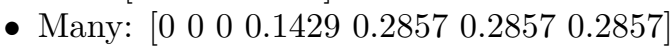

where the second one is derived from the expression "As many as possible".

One of easiest ways to measure the quality of the obtained results is to calculate the percentage of well (or wrong) classief pixels in the image. In Table 1 we show the percentage of pixels well classified with our method, using just one grouping expression (without combination). Any column corresponds to one expression, while every row represents one of the ten images. The last row shows the average percentage over all images.

Based on the percentage of well classified pixels, we can see that the results obtained by expressions $G_{G} 4, G_{G} 5$ and $G_{G} 7$ are, in general, worse than the ones obtained by the four other expressions. Based on this result, we consider that these expressions are not suitable for this kind of images. In this sense, if we want to segment more similar images, we would not use them. But a priori we did not know which expressions are the most suitable, so all of them are in the experiment.

In Table 2 we show, in the same way as Table 1 , the percentages obtained by different combinations of grouping functions.

We can observe that the best results are obtained when we use weighting vectors with medium values of orness. In this sense, they ignore the results given by the most extreme grouping expressions and they only consider the intermediate ones. We also notice that if we focus on extreme values, it is better to work with weighting vectors whose orness value is low.

Based on this experiment, we can say that if we do not know a priori which grouping expressions 


\begin{tabular}{cccccccc}
\hline & $G_{G} 1$ & $G_{G} 2$ & $G_{G} 3$ & $G_{G} 4$ & $G_{G} 5$ & $G_{G} 6$ & $G_{G} 7$ \\
\hline Image 1 & 97.53 & 97.15 & 97.15 & 96.93 & 96.43 & $\mathbf{9 7 . 8 9}$ & 96.93 \\
Image 2 & 97.75 & 97.25 & 97.25 & 96.73 & 96.14 & $\mathbf{9 7 . 9 1}$ & 96.85 \\
Image 3 & $\mathbf{9 8 . 2 0}$ & 98.18 & 98.18 & 97.32 & 96.50 & 97.94 & 97.45 \\
Image 4 & $\mathbf{9 7 . 5 3}$ & 97.40 & 97.40 & 96.64 & 95.73 & 97.49 & 96.75 \\
Image 5 & 97.60 & $\mathbf{9 7 . 6 7}$ & $\mathbf{9 7 . 6 7}$ & 96.54 & 95.21 & 97.41 & 96.80 \\
Image 6 & 97.67 & $\mathbf{9 7 . 7 0}$ & $\mathbf{9 7 . 7 0}$ & 96.61 & 94.90 & 97.38 & 96.93 \\
Image 7 & 97.48 & $\mathbf{9 7 . 6 0}$ & 97.56 & 96.06 & 94.43 & 97.11 & 96.55 \\
Image 8 & 97.83 & $\mathbf{9 7 . 9 4}$ & $\mathbf{9 7 . 9 4}$ & 96.15 & 94.43 & 97.15 & 96.50 \\
Image 9 & $\mathbf{9 6 . 9 3}$ & 96.67 & 96.67 & 94.70 & 93.08 & 96.92 & 94.99 \\
Image 10 & 96.20 & 96.20 & 96.20 & 94.46 & 93.13 & $\mathbf{9 6 . 5 3}$ & 9472 \\
\hline Average & $\mathbf{9 7 . 4 7}$ & 97.38 & 97.37 & 96.21 & 95.00 & 97.37 & 96.45 \\
\hline
\end{tabular}

Table 1: Percentage of well classified pixels obtained by our proposal using just one grouping function. Each column represents one grouping expression while each row is associated with an image. The last row is the average percentage of all images. In bold we show the best result for any image.

\begin{tabular}{c|c|cc|cc|cc}
\hline & Equal & Max & Half & Med & Most & Min & Many \\
\hline Image 1 & 97.42 & 96.43 & 97.15 & 97.53 & 97.15 & $\mathbf{9 7 . 8 9}$ & 97.42 \\
Image 2 & 97.61 & 96.14 & 97.12 & 97.75 & 97.25 & $\mathbf{9 7 . 9 1}$ & 97.61 \\
Image 3 & 98.25 & 96.50 & 98.03 & 98.20 & $\mathbf{9 8 . 2 8}$ & 97.94 & 98.25 \\
Image 4 & 97.56 & 95.73 & 97.20 & 97.53 & 97.40 & 97.53 & $\mathbf{9 7 . 5 7}$ \\
Image 5 & 97.64 & 95.21 & 97.29 & 97.60 & $\mathbf{9 7 . 6 7}$ & 97.41 & 97.60 \\
Image 6 & 97.67 & 94.90 & 97.35 & 97.67 & $\mathbf{9 7 . 7 0}$ & 97.38 & 97.67 \\
Image 7 & 97.56 & 94.43 & 96.86 & 97.48 & $\mathbf{9 7 . 6 0}$ & 97.21 & 97.21 \\
Image 8 & 97.92 & 94.43 & 96.97 & 97.83 & $\mathbf{9 7 . 9 4}$ & 97.15 & 97.68 \\
Image 9 & 96.67 & 93.08 & 94.99 & 96.93 & 96.50 & 96.92 & $\mathbf{9 7 . 0 1}$ \\
Image 10 & 96.20 & 93.13 & 94.95 & 96.20 & 96.20 & $\mathbf{9 6 . 5 3}$ & 96.33 \\
\hline Average & 97.45 & 95.00 & 96.79 & $\mathbf{9 7 . 4 7}$ & 97.37 & 97.39 & 97.44 \\
\hline
\end{tabular}

Table 2: Percentage of well classified pixels obtained by our proposal using different weighting vectors for the combination of grouping functions. Each column represents one weighting vector while each row is associated with an image. The last row is the average percentage of all images. In bold we show the best result for any image. 

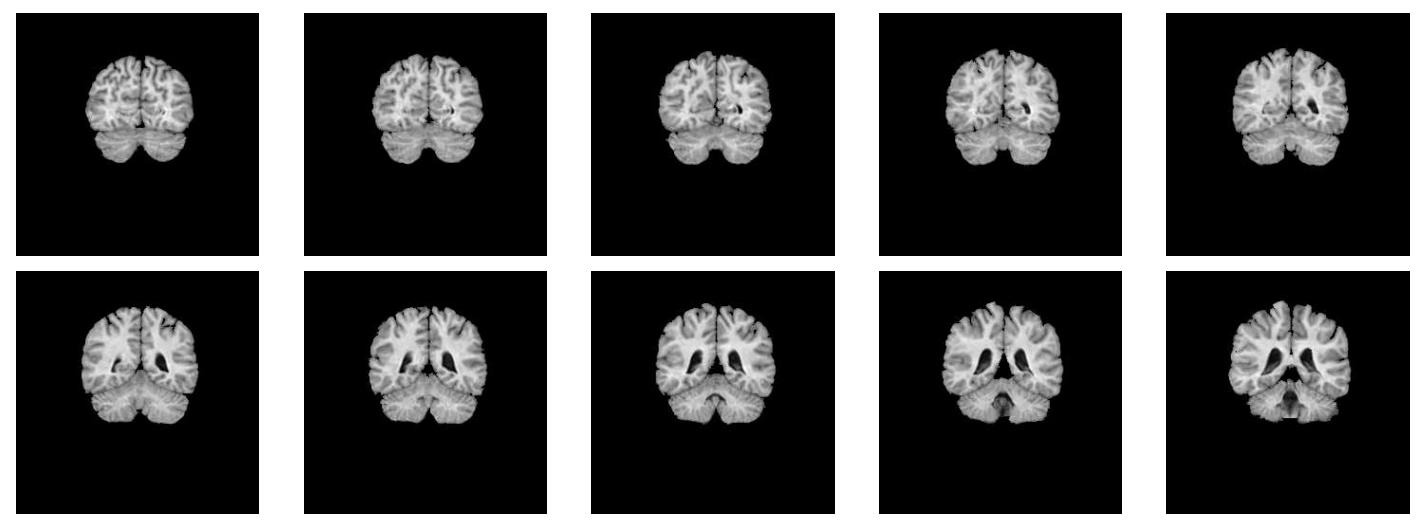

Figure 1: Original images.
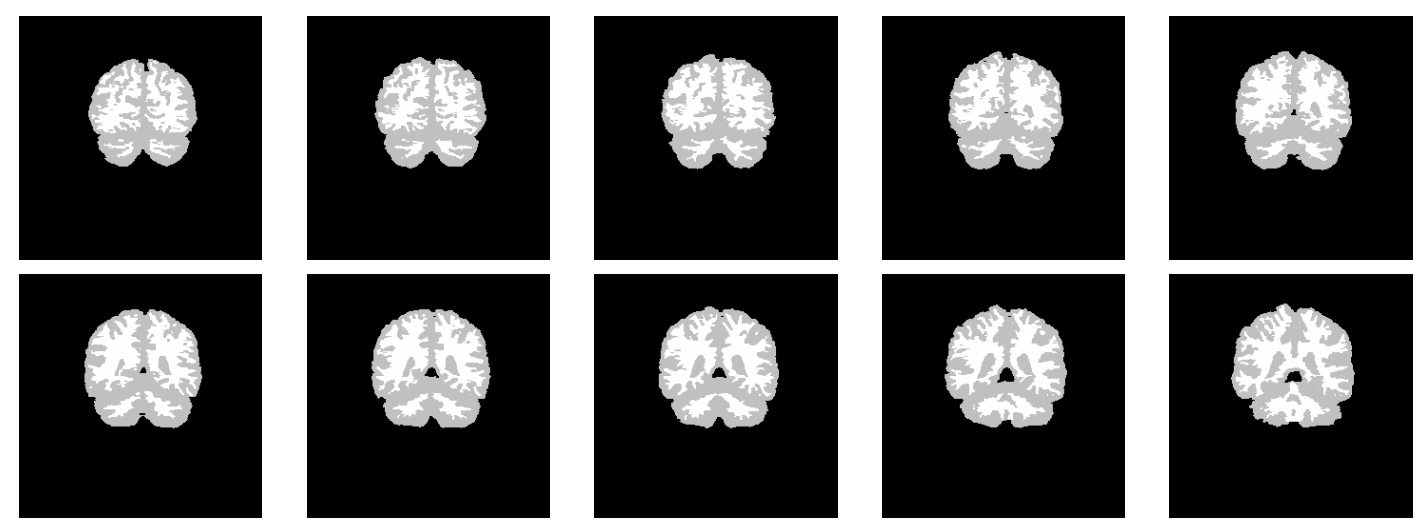

Figure 2: Ideal handmade segmentations.

are suitable for an image (or a specific kind of images) we should use the consensus between several of them by means of weighting vectors with a medium value of orness. We advise to use for example the weighting vector derived from the linguistic quantifier "most". In Figure 3 we show the segmentations obtained by our method for every image.

To finish this experiment, we compare the results obtained by our method with the ones obtained with Otsu [11], which is one of the most used thresholding algorithms (see Table 3). We also compare them with the best possible threshold for every image, it means, the best result that can be achieved by thresholding algorithms over these images.

As we can see from the results we obtain, our method outperforms the one of Otsu. One of the things we want to highlight is that the result we obtain is almost the best we can obtain by thresholding algorithms (only $0.3 \%$ of difference in average, and exactly the best result in 2 of the 10 images).

\section{Conclusions}

In this work we have presented a thresholding algorithm for greyscale images based on grouping functions. These functions, applied to our problem, measure the belongingness of a greylevel intensity

\begin{tabular}{ccc}
\hline Group. & Otsu & Ideal \\
\hline 97.15 & 96.82 & 97.92 \\
97.25 & 96.58 & 97.95 \\
98.28 & 97.45 & 98.28 \\
97.40 & 96.64 & 97.57 \\
97.67 & 96.80 & 97.67 \\
97.70 & 96.13 & 97.72 \\
97.60 & 95.44 & 97.61 \\
97.94 & 95.24 & 97.95 \\
96.50 & 92.35 & 97.03 \\
96.20 & 93.52 & 96.55 \\
\hline 97.37 & 95.70 & 97.63 \\
\hline
\end{tabular}

Table 3: Percentage of well classified pixels obtained by our proposal, Otsu and the ideal threshold for each image. Each row is associated with every image while the last row is the average percentage of all images. 

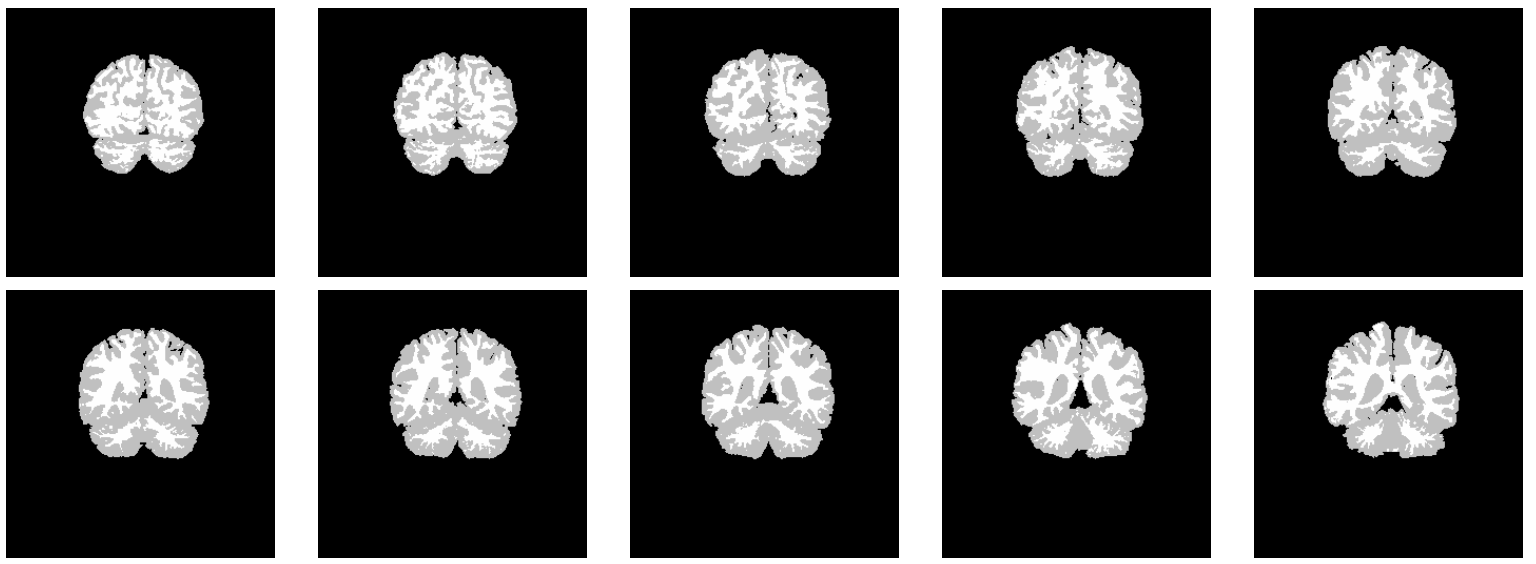

Figure 3: Obtained segmentations by our algorithm using the linguistic quantifier "most" to create the weighting vector.

to the background or to the object of the image. In this way, we choose the threshold associated with the highest grouping value to segment the image. One of the advantages of our proposal is avoiding the selection of a suitable grouping function for each image, by means of a convex combination of several of them. We have carried out a study on how to choose the weights for this combination.

\section{Acknowledgment}

This work was partially supported by the Research Services of the Universidad Publica de Navarra, the National Science Foundation of Spain, references TIN2010-15055 and TIN201129520, the European Regional Development Fund in the IT4Innovations Centre of Excellence project (CZ.1.05/1.1.00/02.0070) and "Strenthening research teams at the University of Ostrava" (CZ.1.07/2.3.00/30.0010).

\section{References}

[1] C. Alsina, M. Frank, and B. Schweizer, Associative functions. Triangular Norms and Copulas, World Scientific, Singapore (2006).

[2] G. Beliakov, A. Pradera, T. Calvo: Aggregation Functions: A Guide for Practitioners. Studies in Fuzziness and Soft Computing 221, 2007.

[3] H. Bustince, E. Barrenechea, M. Pagola: Restricted equivalence functions. Fuzzy Sets and Systems. 157, pp. 2333-2346, 2006.

[4] H. Bustince, E. Barrenechea, M. Pagola: Image thresholding using restricted equivalence functions and maximizing the measure of similarity. Fuzzy Sets and Systems 158, pp. 496-516, 2007.

[5] H. Bustince, J. Fernandez, R. Mesiar, J. Montero, R. Orduna: Overlap functions. Nonlinear Analysis - Theory, Methods \& Applications. 78, pp. 1488-1499, 2010.

[6] H. Bustince, M. Pagola, R. Mesiar, E. Hullermeier, F. Herrera: Grouping, overlap and generalizad bi-entropic functions for fuzzy modelling of pairwise comparisons. IEEE Transactions on Fuzzy Systems 20 (3) pp. 405-415, 2012.

[7] B. Caldairou, N. Passat, P. Habas, C. Studholme, and F. Rousseau, A non-local fuzzy segmentation method: Application to brain MRI, Pattern Recognition, 44 (9) (2011) 19161927.

[8] L.K. Huang, M.J.J. Wang: Image thresholding by minimizing the measure of fuzziness. Pattern Recognition 28, pp. 41-51, 1995.

[9] E.P. Klement, R. Mesiar and E. Pap, Triangular Norms, Trends in Logic. Studia Logica Library, Vol. 8, Kluwer Academic Publishers, Dordrecht, (2000).

[10] A. Mayer and H. Greenspan, An Adaptive Mean-Shift Framework for MRI Brain Segmentation, IEEE Transactions on Medical Imaging, 28 (8) (2009) 105-118.

[11] N. Otsu: A threshold selection method from gray level histograms. IEEE Transactions on Systems, Man and Cybernetics. 9, pp. 62-66, 1979.

[12] P.K. Sahoo, S. Soltani, A.K.C. Wong, Y.C. Chen: A survey of thresholding techniques. Computer Vision Graphics and Image Processing 41, pp. 233-260, 1988.

[13] M. Sezgin, B. Sankur: Survey over image thresholding techniques and quantitative performance evaluation. Journal of Electronic Imaging 13, pp. 146-168, 2004.

[14] E. Trillas: Sobre funciones de negación en la teoría de conjuntos difusos. Stochastica III 1, pp. 47-59, 1979.

[15] R.R. Yager: On ordered weighted averaging aggregation operators in multicriteria decisionmaking. IEEE Transantions on Systems, Man and Cybernetics 18, pp. 183-190, 1988.

[16] R.R. Yager: Quantifier Guided Aggregation Using OWA Operators. International Journal of Intelligent Systems 11, pp. 49-73, 1996. 